\title{
Waist circumference as a predictor of mortality in peritoneal dialysis patients: a follow-up study of 48 months
}

\author{
Ana Catarina M. Castro ${ }^{1}$, Ana Paula Bazanelli ${ }^{1}$, Fabiana B. Nerbass ${ }^{2}$, Lilian Cuppari ${ }^{1,3}$ and \\ Maria A. Kamimura ${ }^{1,3 *}$ \\ ${ }^{1}$ Nutrition Program, Federal University of São Paulo, São Paulo 04038-002, Brazil \\ ${ }^{2}$ Division of Nephrology, Nutrition Department, Pro-rim Foundation, Joinville 80215-901, Brazil \\ ${ }^{3}$ Division of Nephrology, Federal University of São Paulo, São Paulo 04038-002, Brazil \\ (Submitted 1 April 2016 - Final revision received 11 April 2017 - Accepted 28 April 2017-First published online 6 June 2017)
}

\section{Abstract}

Body-fat gain is a common finding among peritoneal dialysis (PD) patients, and the accumulation of adipose tissue occurs predominantly in the abdominal area. Waist circumference (WC) is a reliable marker of abdominal obesity and its association with worse outcomes has been demonstrated in non-dialysis and haemodialysis patients. We aimed at investigating whether WC measurements as well as the changes over time in WC were able to predict mortality in PD patients. This prospective study included 109 patients undergoing PD ( $57 \%$ male, age 52 (sD 16) years, $32 \%$ diabetics, $48 \% \mathrm{BMI} \geq 25 \mathrm{~kg} / \mathrm{m}^{2}$ ). WC was measured at the umbilicus level (empty abdominal cavity), and values $>88 \mathrm{~cm}$ for women and $>102 \mathrm{~cm}$ for men were considered high. Nutritional status and laboratory parameters were also evaluated. WC was measured at baseline and after 6 months, and mortality was registered during a period of 48 months. High WC was observed in $55 \%$ of women and in $23 \%$ of men at baseline. After 6 months, $61 \%$ of the patients showed an increased WC. At the end of the study, twenty-seven deaths were registered. A significant increase in WC was observed only in the non-survivor group. In the Cox regression analysis adjusting for sex, age, duration on dialysis, diabetes, BMI, serum albumin and C-reactive protein, high WC at baseline as well as the 6-month increase in WC were independently associated with mortality. This study demonstrated that a high WC and the increase over time in WC were both predictors of mortality in PD patients.

Key words: Waist circumference: Abdominal obesity: Peritoneal dialysis: Chronic kidney disease: Mortality

The prevalence of abdominal obesity has increased substantially worldwide, in parallel with the increase of overall body adiposity ${ }^{(1)}$. The deleterious effects of abdominal fat accumulation in the general population have been well recognised, and there is increasing evidence of its harmful effect on cardiometabolic abnormalities among patients with chronic kidney disease (CKD) as well ${ }^{(2-5)}$. In the past, studies have reported a relationship between abdominal obesity and mortality among non-dialysis and haemodialysis patients ${ }^{(6-10)}$. Body-fat gain is a common finding among peritoneal dialysis (PD) patients, particularly after commencing dialysis therapy ${ }^{(11-16)}$. Further, there is evidence that the accumulation of body fat occurs predominantly in the abdominal area ${ }^{(17,18)}$. However, the impact of abdominal obesity on clinical outcomes has been scarcely investigated among patients undergoing $\mathrm{PD}^{(19,20)}$.

Waist circumference (WC) is a simple and reliable marker of abdominal fat. In PD patients, some aspects related to the therapy, such as abdominal distension, presence of a catheter and frequent hernia, raised questions regarding the usefulness of WC as a surrogate marker of abdominal adiposity ${ }^{(21)}$. However, when the reproducibility of WC was tested in a recent analysis, WC proved to be a reliable marker of abdominal adiposity in patients undergoing $\mathrm{PD}^{(22)}$. In addition, available data have demonstrated the use of WC as predictor of adipokines and cardiovascular complications $^{(23,24)}$. Here, we aimed to investigate whether WC measurements at baseline and, particularly, whether 6-month changes in WC were able to predict mortality in PD patients during the 48 months of follow-up.

\section{Methods}

Patients

A total of 109 patients undergoing PD (seventy-two on automated PD and thirty-seven on continuous ambulatory PD) were recruited from the Dialysis Unit of the Nephrology Division of the Federal University of São Paulo, Brazil. Only patients older than 18 years, undergoing PD for $>3$ months, free of peritonitis for at least 3 months, and with no catabolic condition were included in the study. Exclusion criteria were treatment with

\footnotetext{
Abbreviations: CKD, chronic kidney disease; PD, peritoneal dialysis; WC, waist circumference.
} 
corticosteroid or immunosuppressive drugs, presence of ascites, severe hernia or malignant disease.

Written informed consent was obtained from each patient. The study was approved by the Ethics and Research Committee of the Federal University of São Paulo.

\section{Study design and protocol}

In this prospective study, WC was measured at baseline and at 6 months. Nutritional status and laboratory parameters were also evaluated. Mortality was recorded over 48 months.

\section{Anthropometry and waist circumference}

BMI was calculated as body weight divided by squared height ${ }^{(25)}$. Measurements of WC were taken when the abdominal cavity was empty. WC was measured at the umbilicus level using a flexible plastic tape measure, while patients were standing with their weight equally distributed on both feet and had their heads facing forward. The mean of three measures was considered for analyses. All WC measurements were performed by the same trained observer.

Values $>88 \mathrm{~cm}$ for women and $>102 \mathrm{~cm}$ for men were considered to be indicative of high risk, in accordance with the Third Report of the National Cholesterol Education Program Expert Panel on Detection, Evaluation and Treatment of High Blood Cholesterol in Adults (Adults Treatment Panel III) (NCEP-ATPIII) $^{(26)}$

\section{Laboratory parameters}

Blood samples were drawn after an overnight fast of $12 \mathrm{~h}$ for assessment of serum levels of glucose, albumin (bromocresol green method) and high-sensitive C-reactive protein (immunochemiluminescence). Patients were instructed to collect the 24-h dialysate and urine samples for weekly measurements of total $K_{t} / V_{\text {urea }}$ and weekly peritoneal creatinine clearance. Presence of residual renal function was considered when urinary volume was $>200 \mathrm{ml} / 24 \mathrm{~h}$.

\section{Statistical analyses}

Data are expressed as means and standard deviations for normally distributed variables and as medians and interquartile ranges for skewed variables. Paired or independent Student's $t$ test was applied, as appropriate. Pearson's correlation test was used to evaluate the association between WC and the studied variables. Cox's regression analysis was applied to investigate whether high WC at baseline and the 6-month changes in WC were predictors of mortality. The variables that significantly affected survival in the univariate analyses, or those known to affect survival according to literature, such as diabetes, inflammation, albumin and BMI, were considered in the multivariate models. In order to respect the proportion of covariates per outcome, we demonstrated models by including different covariates step-by-step. Hazard ratios and $95 \%$ CI were calculated. Differences with $P<0.05$ were considered statistically significant. The analyses were performed using SPSS software, version 18.0 (SPSS Inc.)

\section{Results}

The mean age of the studied patients was 52 (SD 16) years and the median duration on PD was 12 (range 3-104) months. A majority of the patients were male (57\%), 32\% were diabetics, $64 \%$ had residual renal function and $48 \%$ had $\mathrm{BMI} \geq 25 \mathrm{~kg} / \mathrm{m}^{2}$. At baseline, high WC was observed in $55 \%$ of women and in $23 \%$ of men $(P<0.001)$. Patients with higher WC did not differ from those with lower WC in regards to demographic and clinical characteristics. Overall, WC increased from $92 \cdot 6$ (SD 11.9) to $94 \cdot 1$ $(\mathrm{sD} 12.4) \mathrm{cm}(P=0.006)$ after 6 months. No other changes were observed during this period.

All through the 48 months of the study period, twenty-seven deaths were recorded. The causes of the death were CVD ( $n$ 12), sepsis ( $n$ 11), infection ( $n$ 2), multiple organ failure $(n 1)$ and respiratory failure $(n 1)$. The baseline characteristics of the survivor and non-survivor groups are demonstrated in Table 1.

Table 1. Characteristics of the patients at baseline* (Mean values and standard deviations; numbers and percentages; medians and interquartile ranges (IQR))

\begin{tabular}{|c|c|c|c|c|c|}
\hline & \multicolumn{2}{|c|}{$\begin{array}{c}\text { Survivors } \\
(n 82)\end{array}$} & \multicolumn{2}{|c|}{$\begin{array}{c}\text { Non-survivors } \\
(n 27)\end{array}$} & \multirow[b]{2}{*}{$P$} \\
\hline & $n$ & $\%$ & $n$ & $\%$ & \\
\hline \multicolumn{5}{|l|}{ Age (years) } & $<0.01$ \\
\hline Mean & \multicolumn{2}{|c|}{$49 \cdot 5$} & \multicolumn{2}{|c|}{$60 \cdot 2$} & \\
\hline $\mathrm{SD}$ & \multicolumn{2}{|c|}{$15 \cdot 2$} & \multicolumn{2}{|c|}{$17 \cdot 1$} & \\
\hline Male & 43 & 52.4 & 19 & $70 \cdot 4$ & $0 \cdot 10$ \\
\hline Diabetes & 23 & 28 & 12 & $44 \cdot 4$ & 0.11 \\
\hline Residual renal function & 53 & 65.4 & 16 & $59 \cdot 3$ & 0.56 \\
\hline APD & 58 & $70 \cdot 7$ & 14 & 51.9 & 0.07 \\
\hline \multicolumn{6}{|l|}{ Peritoneal equilibrium test (\%) } \\
\hline High transport & \multicolumn{2}{|c|}{$4 \cdot 8$} & \multicolumn{2}{|c|}{5} & \\
\hline High-average transport & \multicolumn{2}{|c|}{$25 \cdot 4$} & \multicolumn{2}{|c|}{35} & \\
\hline Low-average transport & \multicolumn{2}{|c|}{$57 \cdot 1$} & \multicolumn{2}{|c|}{35} & 0.33 \\
\hline Low transport & \multicolumn{2}{|c|}{$12 \cdot 7$} & \multicolumn{2}{|c|}{25} & \\
\hline Previous haemodialysis (\%) & \multicolumn{2}{|c|}{$25 \cdot 6$} & \multicolumn{2}{|c|}{$33 \cdot 3$} & 0.19 \\
\hline Peritonitis (\%) & \multicolumn{2}{|c|}{$40 \cdot 3$} & \multicolumn{2}{|c|}{37} & 0.43 \\
\hline BMI $\left(\mathrm{kg} / \mathrm{m}^{2}\right)$ & & & & & 0.43 \\
\hline Mean & \multicolumn{2}{|c|}{24.9} & \multicolumn{2}{|c|}{$25 \cdot 6$} & \\
\hline SD & \multicolumn{2}{|c|}{$4 \cdot 2$} & \multicolumn{2}{|c|}{$4 \cdot 3$} & \\
\hline $\mathrm{BMI} \geq 25 \mathrm{~kg} / \mathrm{m}^{2}$ & 37 & $45 \cdot 1$ & 15 & $55 \cdot 6$ & 0.35 \\
\hline \multicolumn{5}{|c|}{ Length on peritoneal dialysis (months) } & $0 \cdot 10$ \\
\hline Median & & & & & \\
\hline IQR & & & & & \\
\hline Serum creatinine $(\mu \mathrm{mol} / \mathrm{l})$ & & & & & 0.17 \\
\hline Median & & & & & \\
\hline IQR & $548 \cdot 1$ & $45 \cdot 9$ & 495 & $36 \cdot 8$ & \\
\hline Serum albumin $(g / l)$ & & & & & 0.19 \\
\hline Median & & & & & \\
\hline IQR & $36 \cdot 0$ & & 34 & $9 \cdot 0$ & \\
\hline Serum albumin $<35 \mathrm{~g} / \mathrm{l}$ & 19 & $23 \cdot 2$ & 10 & 37 & 0.16 \\
\hline C-reactive protein (mg/l) & & & & & $0 \cdot 24$ \\
\hline Median & & & & & \\
\hline IQR & $1 \cdot 0$ & & 1.0 & & \\
\hline C-reactive protein $>5 \mathrm{mg} / \mathrm{l}$ & 34 & 41.5 & 14 & 51.9 & 0.37 \\
\hline Total clearance (litres/week) & & & & & $0 \cdot 18$ \\
\hline Median & & & & & \\
\hline IQR & $43 \cdot 9$ & & 53 & 2.4 & \\
\hline Total $K_{t} / V_{\text {urea }}$ & & & & & 1.00 \\
\hline Median & & & & & \\
\hline IQR & $1 \cdot 8$ & & & & \\
\hline
\end{tabular}

APD, automated peritoneal dialysis. * Student's $t$ test. 


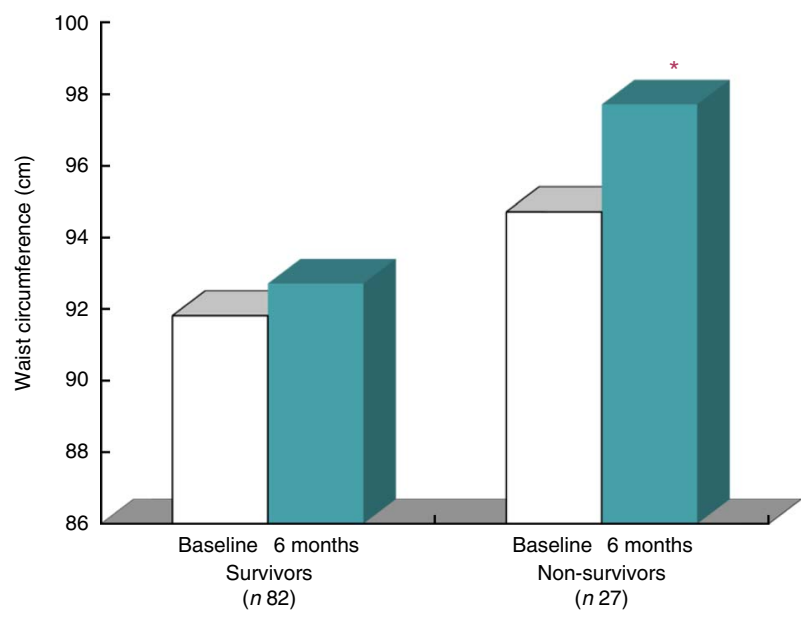

Fig. 1. Waist circumference at baseline and after 6 months in surviving and non-surviving peritoneal dialysis patients. ${ }^{*} P<0.05$ (independent Student's $t$ test).

Table 2. Cox's regression analysis for high waist circumference at baseline

(Hazard ratios and $95 \%$ confidence intervals)

\begin{tabular}{lccc}
\hline & Hazard ratios & $95 \% \mathrm{Cl}$ & $P$ \\
\hline Model 1: sex + age $>60$ years & 5.3 & $1.4,20.5$ & 0.02 \\
Model 2: model 1 + DM + length PD & 6.8 & $1.6,27.9$ & 0.01 \\
Model 3: model 2 + albumin $<35 \mathrm{~g} / /$ & 7.1 & $1.8,29.6$ & 0.01 \\
Model 4: model 3 + BMl $\geq 25 \mathrm{~kg} / \mathrm{m}^{2}$ & 6.9 & $1.2,40.9$ & 0.03 \\
Model 5: model 4+CRP $>5.0 \mathrm{mg} / \mathrm{l}$ & 7.1 & $1.2,43.2$ & 0.03 \\
\hline
\end{tabular}

DM, diabetes mellitus; PD, peritoneal dialysis; CRP, C-reactive protein.

Table 3. Cox regression analysis for 6-month increase in waist circumference

(Hazard ratios and $95 \%$ confidence intervals)

\begin{tabular}{lccc}
\hline & Hazard ratios & \multicolumn{1}{c}{$95 \% \mathrm{Cl}$} & $P$ \\
\hline Model 1: sex + age >60 years & 2.9 & $0.9,9.2$ & 0.06 \\
Model 2: model 1 + DM + length PD & 3.4 & $1.0,11.6$ & 0.05 \\
Model 3: model 2 + albumin change & 4.9 & $1.1,22.4$ & 0.04 \\
Model 4: model 3 + BMI $\geq 25 \mathrm{~kg} / \mathrm{m}^{2}$ & 5.1 & $1.1,22.8$ & 0.03 \\
Model 5: model 4+ CRP change & 13.6 & $2.2,83.8$ & 0.01
\end{tabular}

DM, diabetes mellitus; PD, peritoneal dialysis; CRP, C-reactive protein.

As can be seen, the non-survivor group was older and no other differences were found between the groups. Fig. 1 depicts the WC measurements at baseline and after 6 months in the survivor and non-survivor groups. Of note, a significant increase in WC was observed only in the non-survivor group. In the Cox regression analysis adjusting for sex, age, time on dialysis, presence of diabetes, BMI, serum albumin and C-reactive protein, high WC at baseline (Table 2) and the 6-month increase in WC (Table 3) were independently associated with mortality in PD patients. The incidence of peritonitis, changes in residual renal function, volume status, and PD modality were not associated with mortality in our study.

\section{Discussion}

In the present study, high WC was a predictor of mortality in PD patients. And, more importantly, we showed that changes over time in WC were associated with higher mortality in these patients. The current finding is relevant as available data show that PD patients accumulate body fat over time, particularly in the abdominal area ${ }^{(17,18)}$. In fact, Fernstrom et al. $^{(17)}$ showed that visceral fat, measured by computed tomography, increased substantially in a sample of incident PD patients followed up for 15 months. In addition, a subsequent investigation including sixty patients demonstrated that both visceral and subcutaneous fat increased during the first 6 months on PD therapy ${ }^{(18)}$.

WC is the most simple and inexpensive surrogate of abdominal adiposity, and its reliability against 'gold standard' methods has been well recognised in the general population ${ }^{(27-30)}$. In the CKD population, Sanches et $a l .{ }^{(31)}$ showed in a cross-sectional study including 122 non-dialysed patients that WC was strongly correlated with visceral as well as with subcutaneous fat as assessed by computed tomography. Moreover, the authors found that WC was associated with cardiovascular risk factors in the same manner as was visceral fat. In a subsequent study including the same cohort, Velludo et al. ${ }^{(32)}$ found an agreement between changes over time in WC and visceral fat. The use of WC in PD patients has been avoided by some researchers ${ }^{(21,33)}$ owing to some aspects related to the PD therapy, such as abdominal distension due to fluid infusion into the peritoneal cavity, presence of catheter and hernia, which could compromise the accuracy of WC measurements ${ }^{(34)}$. However, taking into consideration some features such as emptied abdominal cavity, absence of severe hernia and, finally, standardisation and training of the WC measurement technique, WC was shown to be a reliable marker of abdominal adiposity among PD patients ${ }^{(22)}$.

The association of WC with mortality, however, has been scarcely reported in a PD population. A Korean retrospective study, which explored this relationship, followed eighty-four patients on PD for 53.2 (SD 34.4) months found that both all-cause and cardiovascular-cause mortality were not associated with the presence of abdominal obesity assessed by WC. However, the cut-off points used for classification were different from ours $(90 \mathrm{~cm} \text { for males and } 80 \mathrm{~cm} \text { for females })^{(19)}$. In haemodialysis patients, Postorino et al. ${ }^{(6)}$ have demonstrated a direct association between WC and all-cause and cardiovascular death. Kramer et $a l .{ }^{(7)}$ reported that WC was able to determine the mortality risk associated with obesity in a study including a large sample of non-dialysis patients. By using waist:hip ratio $(\mathrm{W} / \mathrm{H})$ as a centralobesity marker, Su et al. ${ }^{(35)}$ found that a high $\mathrm{W} / \mathrm{H}$ was associated with increased cardiovascular events and all-cause mortality in a small number of PD patients followed up for $3 \cdot 1$ years.

In line with the previous studies in CKD, we demonstrated here that the simple anthropometric measure of WC was a predictor of mortality in PD patients. This is the first prospective study to demonstrate the association of high WC with mortality in PD patients, and the first one to establish the relationship between changes over time in abdominal obesity with mortality in a CKD population.

The exact mechanisms associated with high abdominal adiposity and mortality in these particular patients are not well established. A potential reason could be related to the fact that increased abdominal adiposity is strongly associated with insulin resistance, dyslipidaemia and systemic inflammation, which play essential roles in the pathogenesis of CVD in patients with 
$\mathrm{CKD}^{(2-5)}$. In fact, there is evidence of increased WC with reduced adiponectin levels among PD patients ${ }^{(23)}$. Moreover, a recent study demonstrated that WC was independently associated with carotid intima media thickness in PD patients ${ }^{(24)}$. Accordingly, cardiovascular complications were the main cause of mortality in the present cohort (44\%).

It has been suggested that subjects with higher WC have a greater death risk than those with normal WC, regardless of BMI values $^{(6)}$. Accordingly, the mortality predictability of WC was observed in the present study even after adjustment for BMI. Thus, in agreement with other investigators, we highlight that caution is needed when interpreting the relationship between BMI and mortality in PD patients without taking into account the WC measurement.

The limitation of our study is the relatively small cohort of patients; hence, studies with larger samples are required. Besides, an additional prevalent characteristic of the cohort could be added in order to better explore the impact of other variables on mortality. The lack of 'gold standard' methodologies for abdominal adiposity such as computed tomography or MRI could be another limitation in the current study. However, the validity of WC has been previously demonstrated in cross-sectional as well as prospective studies. As WC cut-off values employed in the current study were based on a non-CKD-specific population (NCEP-ATPIII), studies addressing the optimal WC values linked to mortality in PD patients might provide further insight.

In conclusion, this study demonstrated that high WC as well as the 6-month increase in WC were both predictors of mortality in PD patients. We suggest WC to be incorporated as an important tool in the routine care of patients undergoing PD therapy.

\section{Acknowledgements}

The authors thank Fundação de Amparo à Pesquisa do Estado de São Paulo (FAPESP) and the Oswaldo Ramos Foundation.

A. C. M. C. received a scholarship from Coordenação de Aperfeiçoamento de Pessoal de Nível Superior. This manuscript was supported by FAPESP (Process no. 06/56124-6) and the Oswaldo Ramos Foundation.

A. C. M. C., M. A. K. performed statistical analysis and the interpretation of data. A. C. M. C, M. A. K. and F. B. N. drafted the manuscript. A. P. B. was responsible for data collection and review of the content. F. B. N. and L. C. critically reviewed the paper. L. C. and M. A. K. were responsible for the conception and design and final approval of the study. L. C. obtained funding. M. A. K. supervised the study.

The authors declare that there are no conflicts of interest.

\section{References}

1. Li C, Ford ES, McGuire LC, et al. (2007) Increasing trends in waist circumference and abdominal obesity among US adults. Obesity (Silver Spring) 15, 216-224.

2. Odamaki M, Furuya R, Ohkawa S, et al. (1999) Altered abdominal fat distribution and its association with the serum lipid profile in non-diabetic haemodialysis patients. Nephrol Dial Transplant 14, 2427-2432.

3. Yamauchi T, Kuno T, Takada H, et al. (2003) The impact of visceral fat on multiple risk factors and carotid atherosclerosis in chronic haemodialysis patients. Nephrol Dial Transplant 18, 1842-1847.

4. Gohda T, Gotoh H, Tanimoto M, et al. (2008) Relationship between abdominal fat accumulation and insulin resistance in hemodialysis patients. Hypertens Res 31, 83-88.

5. Kato A, Ishida J, Endo Y, et al. (2010) Association of abdominal visceral adiposity and thigh sarcopenia with changes of arteriosclerosis in haemodialysis patients. Nephrol Dial Transplant 26, 1967-1976.

6. Postorino M, Marino C, Tripepi G, et al. (2009) Abdominal obesity and all-cause and cardiovascular mortality in end-stage renal disease. J Am Coll Cardiol 53, 1265-1272.

7. Kramer H, Shoham D, McClure LA, et al. (2011) Association of waist circumference and body mass index with all-cause mortality in CKD: The REGARDS (Reasons for Geographic and Racial Differences in Stroke) Study. Am J Kidney Dis $\mathbf{5 8}$, $177-185$.

8. Elsayed EF, Tighiouart H, Weiner DE, et al. (2008) Waist-to-hip ratio and body mass index as risk factors for cardiovascular events in CKD. Am J Kidney Dis 52, 49-57.

9. Cordeiro AC, Qureshi AR, Stenvinkel P, et al. (2010) Abdominal fat deposition is associated with increased inflammation, protein-energy wasting and worse outcome in patients undergoing haemodialysis. Nephrol Dial Transplant 25, 562-568.

10. Kamimura MA, Carrero JJ, Canziani MEF, et al. (2013) Visceral obesity assessed by computed tomography predicts cardiovascular events in chronic kidney disease patients. Nutr Metab Cardiovasc Dis 23, 891-897.

11. Stenvinkel P, Lindholm B, Lönnqvist F, et al. (2000) Increases in serum leptin levels during peritoneal dialysis are associated with inflammation and a decrease in lean body mass. J Am Soc Nephrol 11, 1303-1309.

12. Johansson AC, Samuelsson O, Haraldsson B, et al. (1998) Body composition in patients treated with peritoneal dialysis. Nephrol Dial Transplant 13, 1511-1517.

13. Jager KJ, Merkus MP, Huisman RM, et al. (2001) Nutritional status overtime in hemodialysis and peritoneal dialysis. $J \mathrm{Am}$ Soc Nephrol 12, 1272-1279.

14. Soreide R, Dracup B, Svarstad E, et al. (1992) Increased total body fat during PD treatment. Adv Perit Dial 8, 173-176.

15. Heimbürger O, Lönnqvist F, Danielsson A, et al. (1997) Serum immunoreactive leptin concentration and its relation to the body fat content in chronic renal failure. J Am Soc Nephrol $\mathbf{8}$, 1423-1430.

16. Jolly S, Chatatalsingh C, Bargman J, et al. (2001) Excessive weight gain during peritoneal dialysis. Int J Artif Organs 24, 197-202.

17. Fernstrom A, Hylander B, Moritz A, et al. (1998) Increase of intra-abdominal fat in patients treated with continuous ambulatory peritoneal dialysis. Perit Dial Int 18, 166-171.

18. Choi SJ, Kim NR, Hong SA, et al. (2010) Changes in body fat mass in patients after starting peritoneal dialysis. Perit Dial Int 31, 67-73.

19. Jin H, Shin JY, Seung HL, et al. (2015) Abdominal obesity and mortality in continuous ambulatory peritoneal dialysis patients. Electrolyte Blood Press 13, 22-29.

20. Lu Q, Cheng LT, Wang T, et al. (2008) Visceral fat, arterial stiffness, and endothelial function in peritoneal dialysis patients. J Ren Nutr 18, 495-502.

21. Iglesias P \& Diez JJ (2010) Adipose tissue in renal disease: clinical significance and prognostic implications. Nephrol Dial Transplant 25, 2066-2077. 
22. Bazanelli AP, Kamimura MA, Manfredi SR, et al. (2012) Usefulness of waist circumference as a marker of abdominal adiposity in peritoneal dialysis: a cross-sectional and prospective analysis. Nephrol Dial Transplant 27, 790-795.

23. Bazanelli AP, Kamimura MA, Canziani ME, et al. (2013) Waist circumference as a predictor of adiponectin levels in peritoneal dialysis patients: a 12-month follow-up study. Perit Dial Int 33, 182-188.

24. Asicioglu E, Kahveci A, Arikan H, et al. (2013) Waist circumference is associated with carotid intima media thickness in peritoneal dialysis patients. Int Urol Nephrol 45, 1437-1443.

25. Keys A, Fidanza F, Karvonen MJ, et al. (1972) Indices of relative weight and obesity. J Chronic Dis 25, 329-343.

26. National Cholesterol Education Program (NCEP) Expert Panel on Detection, Evaluation, and Treatment of High Blood Cholesterol in Adults (Adult Treatment Panel III) (2002) Third Report of the National Cholesterol Education Program (NCEP) Expert Panel on Detection, Evaluation and Treatment of High Blood Cholesterol in Adults (Adult Treatment Panel III) final report. Circulation 106, 3143-3421.

27. Despres JP, Prud'homme D, Pouliot MC, et al. (1991) Estimation of deep abdominal adipose-tissue accumulation from simple anthropometric measurements in men. Am J Clin Nutr 54, 471-477.

28. Janssen I, Katzmarzyk PT \& Ross R (2002) Body mass index, waist circumference, and health risk: evidence in support of current National Institutes of Health guidelines. Arch Intern Med 162, 2074-2079.

29. Stewart KJ, DeRegis JR, Turner KL, et al. (2003) Usefulness of anthropometrics and dual-energy x-ray absorptiometry for estimating abdominal obesity measured by magnetic resonance imaging in older men and women. J Cardiopulm Rehabil 23, 109-114.

30. Vega GL, Adams-Huet B, Peshock R, et al. (2006) Influence of body fat content and distribution on variation in metabolic risk. J Clin Endocrinol Metab 91, 4459-4466.

31. Sanches FM, Avesani CM, Kamimura MA, et al. (2008) Waist circumference and visceral fat in CKD: a cross-sectional study. Am J Kidney Dis 52, 66-73.

32. Velludo CM, Kamimura MA, Sanches FM, et al. (2010) Prospective evaluation of waist circumference and visceral adipose tissue in patients with chronic kidney disease. Am J Nephrol 31, 104-109.

33. Zhe XW, Zeng J, Tian XK, et al. (2008) Pulse wave velocity is associated with metabolic syndrome components in CAPD patients. Am J Nephrol 28, 641-646.

34. Nurmi J, Korkeila M, Honkanen E, et al. (2010) Effect of peritoneal dialysis on abdominal circumference. Perit Dial Int 30, 215-217.

35. Su WS, Clase CM, Brimble KS, et al. (2010) Waist-to-hip ratio, cardiovascular outcomes, and death in peritoneal dialysis patients. Int J Nephrol 2010, 831243. 